\title{
CONSTRAINTS ON HYDROSTATIC MODELS OF SOFT GAMMA-RAY REPEATERS
}

\author{
M. Coleman Miller \\ University of Chicago, Department of Astronomy and Astrophysics, 5640 South Ellis Avenue, Chicago, IL 60637; miller@gamma.uchicago.edu \\ Received 1995 February 1; accepted 1995 May 15
}

\begin{abstract}
All three soft gamma-ray repeaters (SGRs) are associated with supernova remnants; consequently, models of SGRs involving young neutron stars have attracted close attention. From the distances to the supernova remnants and the observed burst fluxes, the luminosity of a typical burst from an SGR is $\sim 10^{41}-10^{42} \mathrm{ergs} \mathrm{s}^{-1}$; the 1979 March 5 event from SGR 0526-66 had an inferred peak luminosity of $\sim 5 \times 10^{44} \mathrm{ergs} \mathrm{s}^{-1}$. These luminosities are orders of magnitude larger than the Eddington luminosity $L_{\mathrm{E}} \sim 2 \times 10^{38} \mathrm{ergs} \mathrm{s}^{-1}$ for a neutron star.

Many models of SGRs assume that the atmosphere is hydrostatic. To reconcile this requirement with the large luminosities of SGRs, it has been suggested that the neutron stars that produce SGRs have extremely high magnetic fields. Then for a photon with frequency $\omega$ much less than the electron cyclotron frequency $\omega_{c}$, the scattering cross section (and thus the radial radiation force) in the perpendicular polarization mode is decreased by a factor $\sim\left(\omega / \omega_{c}\right)^{2}$ compared to the magnetic field $B=0$ case. It has been assumed that if essentially all of the radiation emerges in the perpendicular mode, then the maximum hydrostatic luminosity rises to $L_{\text {crit }} \sim\left(\omega_{c} / \omega\right)^{2} L_{\mathrm{E}}$. Thus, to allow a hydrostatic atmosphere for a burst luminosity $\sim 10^{42} \mathrm{ergs} \mathrm{s}^{-1}$ and a typical photon energy $\hbar \omega \sim 50$ $\mathrm{keV}$, it has been thought that a magnetic field $B \sim 5 \times 10^{14} \mathrm{G}$ would suffice.

We show that this is not correct. Although most of the luminosity emerges in the perpendicular mode, the fraction $\sim\left(\omega / \omega_{c}\right)$ that scatters into the parallel mode dominates the radiation force. Hence the maximum luminosity is only $L_{\text {crit }} \sim 5\left(\omega_{c} / \omega\right) L_{\mathrm{E}}$. Thus, for a typical SGR burst, the magnetic field must be at least $B \sim 10^{16}$ $\mathrm{G}$ to ensure a hydrostatic atmosphere, and for the March 5 event $B>5 \times 10^{18} \mathrm{G}$ is required. These field strengths are unreasonably high. We therefore suggest that SGRs do not have hydrostatic atmospheres and that models which invoke magnetic confinement are more promising.
\end{abstract}

Subject headings: gamma rays: bursts — magnetic fields — radiative transfer — stars: neutron

\section{INTRODUCTION}

Classical gamma-ray bursts (GRBs) have been studied for more than 20 years, but modeling of GRBs has been hampered by the lack of distance determinations and by the lack of quiescent counterparts. Until recently, the study of soft gamma-ray repeaters (SGRs) (which may be a related phenomenon) faced nearly the same problem because only the source SGR 0526-66, which produced the unusual 1979 March 5 event (Mazets et al. 1979; Mazets \& Golenetskii 1981), had been positionally identified with a known object, the supernova remnant N49 in the Large Magellanic Cloud (Evans et al. 1980). However, both SGR 1806-20 and SGR $1900+14$ have now been associated with supernova remnants as well (Kouveliotou et al. 1993; Kulkarni \& Frail 1993; Kulkarni et al. 1994; Kouveliotou et al. 1994; Hurley et al. 1994; Vasisht et al. 1994). The association of all three SGRs with supernova remnants is almost certainly not a coincidence, so recent models of SGRs have concentrated on processes involving young neutron stars.

The fluxes observed from SGRs, combined with estimates of the distances to the associated supernova remnants, imply that a typical burst from an SGR reaches a luminosity of $\sim 10^{41}$ $10^{42} \mathrm{ergs} \mathrm{s}^{-1}$ for $\sim 0.5 \mathrm{~s}$ (Fenimore, Laros, \& Ulmer 1994). This luminosity exceeds the standard Eddington luminosity $L_{\mathrm{E}} \equiv 1.3 \times 10^{38}\left(M / M_{\odot}\right)$ ergs s${ }^{-1}$, where $M$ is the gravitational mass of the neutron star, by a factor of $\sim 10^{3}-10^{4}$. Thus, if the interaction cross section of the radiation with the plasma in the atmosphere of the neutron star is the Thomson cross section $\sigma_{\mathrm{T}}=6.65 \times 10^{-25} \mathrm{~cm}^{2}$ and the plasma is mostly ionized hydrogen, then in the absence of other effects, the plasma would expand rapidly outward in a fireball. However, the spectra of bursts from SGR 1806-20 seem roughly independent of their luminosity, which is difficult to reconcile with a thermal fireball model (Fenimore et al. 1994; Ulmer 1994).

As a way around this problem, it has been noted (Paczyński 1992; Thompson \& Duncan 1993; Ulmer 1994; Thompson \& Duncan 1995) that a hydrostatic atmosphere may be maintained even at luminosities $L \gg L_{\mathrm{E}}$ if the scattering cross section $\sigma$ is much lower than $\sigma_{\mathrm{T}}$. One way to lower $\sigma$ is to increase the magnetic field because in a field with electron cyclotron frequency $\omega_{c}$, the scattering cross section for a photon of frequency $\omega \ll \omega_{c}$ in the perpendicular mode (defined so that the electric field of the photon is perpendicular to the plane containing the magnetic field and the photon propagation direction) is $\sigma_{\perp} \approx\left(\omega / \omega_{c}\right)^{2} \sigma_{\mathrm{T}}$. Thus, for an isolated particle the radiation force from perpendicular mode photons is a factor $\sim\left(\omega / \omega_{c}\right)^{2}$ less than the radiation force from an equal flux of parallel mode photons. If the energy is released deep in the atmosphere of a neutron star, radiation transfer will put most of the luminosity into the low cross section mode, so in previous treatments it has been assumed implicitly that essentially all of the luminosity will emerge in the perpendicular mode. If this were true, the maximum luminosity for a hydrostatic atmosphere in regions of open field lines would rise to $L_{\text {crit }} \approx\left(\omega_{c} / \omega\right)^{2} L_{\mathrm{E}}$. Since SGR bursts have characteristic photon energies $\hbar \omega \sim 50 \mathrm{keV}$, a magnetic field of $B \sim 5 \times 10^{14} \mathrm{G}$ would allow a hydrostatic atmosphere even for $L=2 \times 10^{42} \mathrm{ergs} \mathrm{s}^{-1}$, thus avoiding the problems associated with fireballs.

Here we show that, although most of the radiation flux does indeed come out in the perpendicular mode, the radiation 


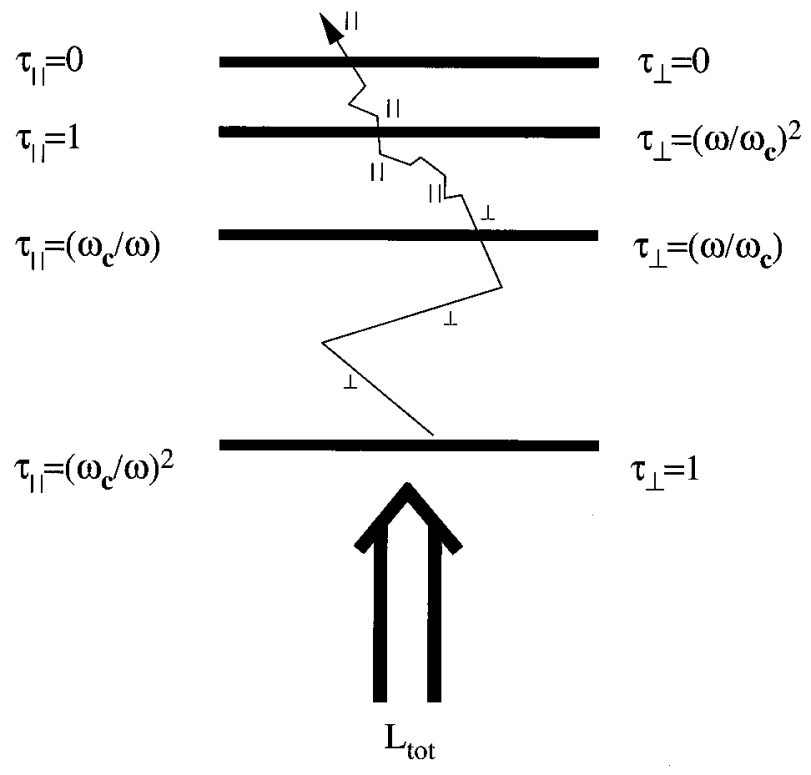

FIG. 1.-Schematic diagram of photon propagation through an atmosphere. A total luminosity $L_{\text {tot }}$ is released deep in the atmosphere at a perpendicular optical depth $\tau_{\perp} \gg 1$. It then scatters many times, occasionally changing polarization, until it finally escapes. The sample photon represented in this figure converted from perpendicular to parallel at $\tau_{\|}<\left(\omega_{c} / \omega\right)$ and was still in the parallel mode when it escaped.

force is dominated by parallel mode photons, which have a scattering cross section $\sigma_{\|} \sim \sigma_{\mathrm{T}}$. As we show to order of magnitude in $\S 2$ and in detail with numerical simulations in $\S 3$, this occurs because a fraction $\sim 0.2\left(\omega / \omega_{c}\right)$ of the perpendicular mode photons convert into the parallel mode and then escape while still in the parallel mode. Therefore, the maximum luminosity is not $L_{\text {crit }} \sim\left(\omega_{c} / \omega\right)^{2} L_{\mathrm{E}}$ but, rather, $L_{\text {crit }} \lesssim 5\left(\omega_{c} / \omega\right) L_{\mathrm{E}}$. Thus, for example, the magnetic field required for a hydrostatic atmosphere with $50 \mathrm{keV}$ photons at $10^{42} \mathrm{ergs} \mathrm{s}^{-1}$ is not $5 \times 10^{14}$ $\mathrm{G}$ but, rather, $B \gtrsim 10^{16} \mathrm{G}$. For the March 5 event, which reached a peak luminosity of $\sim 5 \times 10^{44}$ ergs $\mathrm{s}^{-1}$, a magnetic field $B \gtrsim 5 \times 10^{18} \mathrm{G}$ would be needed. Moreover, although throughout this Letter we make the conservative assumption that the radiation interacts with the matter via scattering only, bound-free absorption and other processes may significantly increase the radiation force (Miller \& Bulik 1995). The true minimum magnetic fields for hydrostatic atmospheres in regions of open field lines are thus even greater than estimated here. Such magnetic fields are improbable, and in $\S 4$ we suggest instead that magnetic confinement, which may be accomplished with much more modest field strengths, is a more promising avenue to explore.

\section{ORDER OF MAGNITUDE TREATMENT}

Before we perform a detailed calculation, we can derive the rough dependence of the critical luminosity on the magnetic field by an order of magnitude estimate. Consider the physical picture shown in Figure 1. In this picture, a total luminosity $L_{\text {tot }}$ is released somewhere deep in the atmosphere, and the photons then propagate upward by scattering. The photons can be divided into two classes, depending on their polarization: either their electric field vector is in the plane of the magnetic field and the photon propagation direction $(\|)$ or the electric field vector is perpendicular to that plane $(\perp)$. In the limit $\omega \ll \omega_{c}$, where the photon frequency is much less than the electron cyclotron frequency, if $\hbar \omega \ll m_{e} c^{2}$ then in a vacuum the scattering cross sections for the two modes are $\sigma_{\|} \sim \sigma_{\mathrm{T}}$ and $\sigma_{\perp} \sim\left(\omega / \omega_{c}\right)^{2} \sigma_{\mathrm{T}}$. In an individual scattering there is a chance that the polarization mode may switch, and the probabilities are (see Herold 1979) $P(\| \rightarrow \perp) \sim\left(\omega / \omega_{c}\right)^{2}$ and $P(\perp \rightarrow \|)=1 / 4$.

Now consider the propagation of the radiation from an initial perpendicular optical depth $\tau_{\perp} \gg 1$. When $\tau_{\perp} \gg 1$, detailed balance guarantees that the number density of perpendicular mode photons is equal to the number density of parallel mode photons. At these high optical depths, most of the flux is transported in the perpendicular mode, but the radiation forces for the two modes are equal to each other. When the radiation gets to $\tau_{\perp} \sim 1$, a significant number of perpendicular mode photons can escape from the atmosphere without scattering, but because at that point the parallel optical depth is $\tau_{\|} \sim\left(\omega_{c} / \omega\right)^{2} \gg 1$, the parallel mode photons are trapped, and most of them eventually scatter into the perpendicular mode. Thus, the fraction of perpendicular mode photons increases with decreasing optical depth, and ultimately the observed photons are almost all in the perpendicular mode.

However, although the escaping luminosity is dominated by the perpendicular mode photons, the radiation force on a test particle at $\tau_{\|}=\tau_{\perp}=0$ is dominated by parallel mode photons. To see this, consider the atmosphere at $\tau_{\perp}=\omega / \omega_{c}$ (so that $\left.\tau_{\|}=\omega_{c} / \omega\right)$. Assume that all the photons are in the perpendicular mode. A fraction $\sim \omega / \omega_{c}$ of those photons will scatter before they reach the surface, and one-fourth of the photons that scatter will switch into the parallel mode. Because $\tau_{\|}=\omega_{c} /$ $\omega$, the newly created parallel photons will scatter $\sim\left(\omega_{c} / \omega\right)^{2}$ times before escaping, and a fraction $\sim\left[1-\left(\omega / \omega_{c}\right)^{2}\right]^{\left(\omega_{c} / \omega\right)^{2}} \sim 1$ / $e$ of those photons will escape while still in the parallel mode. Thus, the luminosity in the parallel mode is approximately

$$
L_{\|} \sim \frac{1}{e} \frac{1}{4}\left(\omega / \omega_{c}\right) L_{\text {tot }} \sim 0.1\left(\omega / \omega_{c}\right) L_{\text {tot }} .
$$

Since $\sigma_{\|} \approx \sigma_{\mathrm{T}}$, the requirement of a hydrostatic atmosphere implies $L_{\|}<L_{\mathrm{E}}$, or

$$
L_{\text {tot }} \lesssim 10\left(\omega_{c} / \omega\right) L_{\mathrm{E}} .
$$

The more detailed calculations presented below show that the coefficient is actually 5 , not 10 .

\section{NUMERICAL RESULTS}

To estimate more precisely the total radiation force, we performed numerical simulations of photons propagating through a neutron star atmosphere. In these simulations we assumed that the photons interacted via scattering only and that the energy of the photons was unchanged by the scattering. We started each photon in the perpendicular mode at $\tau_{\perp}=3$ and followed until it escaped from the atmosphere, and we assumed that the magnetic field direction was parallel to the surface normal. We then computed the radiation force and radial radiation flux for each photon and determined the total force per unit flux.

For each scattering, we calculated the direction of scattering, mean free path, and polarization using the differential cross section derived by Herold (1979), which is valid for low plasma density when $\hbar \omega \ll m_{e} c^{2}$ and $\hbar \omega \ll \hbar \omega_{c}$ :

$$
\frac{d \sigma}{d \Omega^{\prime}}=\frac{1}{4} r_{0}^{2}\left|a\left(s \rightarrow s^{\prime}\right)\right|^{2}
$$


with

$$
\begin{aligned}
& a(\|\rightarrow\|)= 2 \sin \theta \sin \theta^{\prime} \\
&+\cos \theta \cos \theta^{\prime}\left(e^{i\left(\phi-\phi^{\prime}\right)} \frac{\omega}{\omega_{c}}-e^{-i\left(\phi-\phi^{\prime}\right)} \frac{\omega}{\omega_{c}}\right), \\
& a(\perp \rightarrow \perp)=e^{i\left(\phi-\phi^{\prime}\right)} \frac{\omega}{\omega_{c}}-e^{-i\left(\phi-\phi^{\prime}\right)} \frac{\omega}{\omega_{c}}, \\
& a(\| \rightarrow \perp)=-i \cos \theta\left(e^{i\left(\phi-\phi^{\prime}\right)} \frac{\omega}{\omega_{c}}+e^{-i\left(\phi-\phi^{\prime}\right)} \frac{\omega}{\omega_{c}}\right),
\end{aligned}
$$

and

$$
a(\perp \rightarrow \|)=i \cos \theta^{\prime}\left(e^{i\left(\phi-\phi^{\prime}\right)} \frac{\omega}{\omega_{c}}+e^{-i\left(\phi-\phi^{\prime}\right)} \frac{\omega}{\omega_{c}}\right),
$$

where $\theta$ and $\theta^{\prime}$ are, respectively, the initial and final photon propagation directions with respect to the magnetic field; $\phi$ and $\phi^{\prime}$ are the initial and final azimuthal directions; $\Omega^{\prime}$ is the element of solid angle into which the photon is scattering; $s$ and $s^{\prime}$ are the initial and final polarization states; and $r_{0}=2.8 \times 10^{-13} \mathrm{~cm}$ is the classical radius of the electron. If the plasma is dense enough to significantly affect the dielectric tensor, the scattering cross section, and thus the radiation force, is greater than assumed here (see, e.g., Mészáros 1992, p. $89 \mathrm{ff})$.

Since we are ultimately interested in the critical luminosity such that the radial radiation force balances the gravitational force, we need to calculate the radial radiation force for a given radial flux. This force is often assumed to be proportional to the gradient of the radiation pressure. However, $f+\nabla P_{\mathrm{r}}$ if the opacity depends on either the frequency or the incident direction of the photon or if the photon mean free path is large compared to other relevant length scales (Lamb \& Miller 1995); for example, a laser beam in a vacuum exerts a radiation force although the pressure gradient along the beam is negligible. Thus, the radiation force at $\tau \ll 1$ must be calculated with a more general formula.

From Lamb \& Miller (1995), the radial radiation force $f_{\mathrm{rad}}^{\hat{r}}$ on a particle at position $x^{\gamma}$ due to a radiation field with specific intensity $I\left(n, \nu ; x^{\gamma}\right)$ (where $n$ and $\nu$ are the direction and frequency of the radiation) is

$$
f_{\mathrm{rad}}^{\hat{r}}=\frac{1}{c} \int_{0}^{\infty} d \nu \int_{4 \pi} d \Omega I\left(\mathbf{n}, \nu ; x^{\gamma}\right) \sigma^{\hat{\gamma}}(\mathbf{n}, \nu),
$$

where

$$
\sigma^{\hat{r}}(\mathbf{n}, \nu)=\int_{0}^{\infty} d \nu^{\prime} \int_{4 \pi} d \Omega^{\prime} \frac{d \sigma}{d \Omega^{\prime}}\left(\mathbf{n}, \nu ; \mathbf{n}^{\prime}, \nu^{\prime}\right) n^{\hat{r}} .
$$

is the cross section for momentum transfer in the radial direction and the radiation is assumed to scatter into a solid angle $d \Omega^{\prime}$ around the direction $\boldsymbol{n}^{\prime}$. Equation (6) assumes that the photon frequency is unchanged by the scattering and that the differential cross section is symmetric about the radial direction (because we have assumed that the magnetic field is in the radial direction). Similarly, the radial flux at $x^{\gamma}$ is

$$
F\left(n^{\hat{r}} ; x^{\gamma}\right)=c \int_{0}^{\infty} d \nu \int_{4 \pi} d \Omega I\left(\mathbf{n}, \nu ; x^{\gamma}\right) n^{\hat{r}}
$$

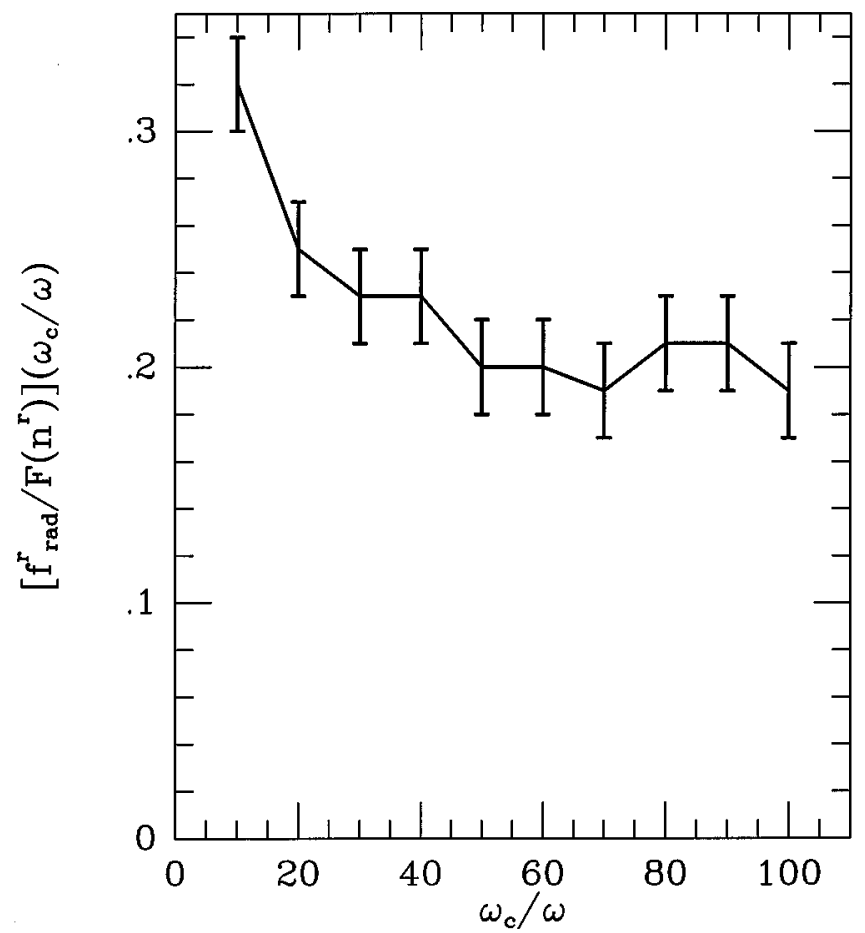

FIG. 2.-Radial force per flux vs. $\omega_{c} / \omega$, the electron cyclotron frequency divided by the photon frequency. The ratio $f_{\text {rad }}^{\hat{r}} / F\left(n^{\hat{r}}\right)$ is multiplied by $\omega_{c} / \omega$ to demonstrate the scaling $f_{\mathrm{rad}}^{\hat{r}} \sim\left(\omega / \omega_{c}\right)$. The statistical error in $\left[f_{\mathrm{rad}}^{\hat{r}} / F\left(n^{\hat{r}}\right)\right]\left(\omega_{c} / \omega\right)$ is $\approx 0.02$ in each case.

(Lamb \& Miller 1995). In the numerical calculations the integrals are replaced by sums over the incident photons. In our case, a photon emerging at an angle $\theta$ with respect to the magnetic field contributes a radial flux proportional to $\cos \theta$ and a radial force proportional to $\sin ^{2} \theta \cos \theta$ if the photon is in the parallel mode versus a radial radiation force $\sim\left(\omega / \omega_{c}\right)^{2}$ $\cos \theta$ if the photon is in the perpendicular mode. Note, however, that the expressions for the radial radiation force are more complicated for arbitrary magnetic field orientations. To estimate the force for a given flux, we propagated 50,000100,000 photons through the atmosphere (depending on the value of $\left.\omega_{c} / \omega\right)$, got the total radial flux and total radial radiation force, and divided the force by the flux. To make the code more efficient, we assumed that photons at $\tau_{\perp}>3$ remained in the perpendicular mode. This is physically justified because the distance traveled in the parallel mode at that depth is negligible, and this approximation makes less than a $1 \%$ difference in the results presented here.

Figure 2 shows the results of these calculations for $\omega_{c} / \omega=10-100$, where we plot the force divided by the flux, $f_{\text {rad }}^{\hat{r}} / F\left(n^{\hat{r}}\right)$, multiplied by $\omega_{c} / \omega$, against $\omega_{c} / \omega$. For $\omega_{c} / \omega \gg 1$, $\left[f_{\text {rad }}^{r} / F\left(n^{r}\right)\right]\left(\omega_{c} / \omega\right)$ approaches $\sim 0.2$ asymptotically. Thus, as indicated by the order-of-magnitude estimate, for $\omega_{c} / \omega \gg 1$ the radiation force for a given luminosity is much greater than has been previously assumed. For example, when $\omega_{c} / \omega=100$, the radiation force is $2 \times 10^{-3}$ times the nonmagnetic radiation force, so that a luminosity of $\sim 10^{41} \mathrm{ergs} \mathrm{s}^{-1}$ could be maintained hydrostatically along open field lines, but $10^{42}$ ergs $\mathrm{s}^{-1}$ would require at least $\omega_{c} / \omega=1000$. 


\section{DISCUSSION AND SUMMARY}

Because models involving unconfined fireballs do not match the spectral behavior of SGRs (Fenimore et al. 1994), attention has focused on ways to confine the burst energy near the surface of the neutron star. It was pointed out by Fenimore et al. (1994) and by Ulmer (1994) that in a strong magnetic field, radiation transfer effects would tend to put most of the emerging luminosity in the low cross section mode (which we have called the perpendicular mode). In the diffusion approximation, they found that only a fraction $\sim\left(\omega / \omega_{c}\right)^{2}$ of the emerging photons would be in the high cross section mode, implying that the maximum luminosity allowed along open field lines for a hydrostatic atmosphere is $L_{\text {crit }} \sim\left(\omega_{c} / \omega\right)^{2} L_{\mathrm{E}}$ and that magnetic fields $\sim 10^{14}-10^{15} \mathrm{G}$ are required to explain typical SGRs.

However, as we have shown here, a fraction $\sim \omega / \omega_{c}$ of perpendicular mode photons convert to parallel mode photons at low optical depths. As a result, the radiation force scales as $\left(\omega / \omega_{c}\right)$, not as $\left(\omega / \omega_{c}\right)^{2}$, so that much higher magnetic fields are required to achieve the same increase in the maximum luminosity. Moreover, several effects not included in this Letter, including vacuum polarization and mode switching, the proton cyclotron resonance, and bound-free absorption, increase the opacity beyond the simple scattering opacity assumed here (Miller \& Bulik 1995). Photon splitting may also increase the fraction of parallel mode photons. These further increase the radiation force, so the field strengths needed for hydrostatic atmospheres are even larger than those estimated in this Letter. Even with the current conservative estimates, for the March 5 event to be gravitationally confined, the magnetic field would have to be so large that the energy in the field would be enough to disrupt the neutron star.

Note that our Letter does not rule out field strengths of $10^{14}-10^{15} \mathrm{G}$. Indeed, many of the arguments listed by Thompson \& Duncan (1995) for such fields may still apply, such as the calculation that a field $B \sim 6 \times 10^{14} \mathrm{G}$ is required to spin down an initially rapidly rotating neutron star to the observed $\sim 8 \mathrm{~s}$ period of the March 5 event within the $\sim 10,000 \mathrm{yr}$ lifetime of the associated diffuse supernova remnant. What we have shown is that even if $B \sim 10^{14}-10^{15} \mathrm{G}$, gravitational forces alone are not sufficient to prevent radiatively accelerated plasma from escaping.

A more promising source of confinement is the magnetic field itself. As shown by Lamb (1982; see also Katz 1982, 1993, $1994)$, in a closed field geometry a thermal plasma of temperature $T$ can be confined by a magnetic field $B>10^{12}(k T / 170$ $\mathrm{keV})^{2} \mathrm{G}$, so for the temperatures $k T \sim 20 \mathrm{keV}$ derived for SGRs, typical pulsar fields of $B \sim 10^{12} \mathrm{G}$ can confine the plasma easily and may be able to suppress any instabilities. Such a model does require that the energy be emitted away from the magnetic poles because the plasma will move rapidly along the field lines and if it can reach a region of weak field at large radius then it may be able to break away. Moreover, if the plasma gets more than $\sim 10^{6} \mathrm{~cm}$ away from the neutron star surface, then the emitting area will increase, and the temperature will drop below what is observed. Hence, for the magnetic confinement model to match the spectral properties of the SGRs, the energy must be emitted within $\sim 1$ rad of the magnetic equator. An asymmetric release of energy may account for the $8 \mathrm{~s}$ period seen in the March 5 event; however, much more work needs to be done on the magnetic confinement models to make them quantitative and to predict, e.g., what observable effects should be expected from emission along open field lines.

In summary, models of SGRs involving strongly magnetized neutron stars are still viable, but gravitation alone cannot confine the plasma against radiation forces, and consequently magnetic confinement models are more realistic.

Tomek Bulik and Carlo Graziani made valuable comments on issues related to strong-field cross sections, and Fred Lamb, Don Lamb, Chris Thompson, and Ed Fenimore helped clarify previous versions of this Letter. This work was supported by NASA grant NAGW 830 and, through the Compton Fellowship Program, by NASA grant NAS 5-28543.

\section{REFERENCES}

Evans, W. D., et al. 1980, ApJ, 237, L7

Fenimore, E. E., Laros, J. G., \& Ulmer, A. 1994, ApJ, 432, 742

Herold, H. 1979, Phys. Rev. D, 19, 2868

Hurley, K., Sommer, M., Kouveliotou, C., Fishman, G., Meegan, C., Cline, T. Boer, M., \& Niel, M. 1994, ApJ, 431, L31 Katz, J. I. 1982, ApJ, 260, 371

1993, in Compton Gamma-Ray Observatory, ed. M. Friedlander,

N. Gehrels, \& D. J. Macomb (New York: AIP), 1090 1994, ApJ, 422, 248

Kouveliotou, C., et al. 1993, Nature, 362, 728

Kouveliotou, C., et al. 1994, Nature, 368, 125

Kulkarni, S. R., \& Frail, D. A. 1993, Nature, 365, 33

Kulkarni, S. R., Frail, D. A., Kassim, N. E., Murakami, T., \& Vasisht, G. 1994,

Nature, 368,129

Lamb, D. Q. 1982, in Gamma-Ray Transients and Related Astrophysical
Phenomena, ed. R. E. Ligenfelter, H. S. Hudson, \& D. M. Worrall (New York: AIP), 249

Lamb, F. K., \& Miller, M. C. 1995, ApJ, 439, 828

Mazets, E. P., \& Golenetskii, S. V. 1981, Ap\&SS, 75, 47

Mazets, E. P., Golenetskii, S. V., Iiyinskii, V. N., Aptekar, R. L., \& Guryan, Y. A. 1979, Nature, 282, 587

Mészáros, P. 1992, High-Energy Radiation from Magnetized Neutron Stars (Chicago: Univ. of Chicago Press)

Miller, M. C., \& Bulik, T. 1995, in preparation

Paczyński, B. 1992, Acta Astron., 42, 145

Thompson, C., \& Duncan, R. C. 1993, ApJ, 408, 194

Ulmer. 1995, MNRAS, in press

Vasisht, G., Kulkarni, S. R., Frail, D. A., \& Greiner, J. 1994, ApJ, 431, L35 